\title{
Mit viel Arbeit und verständnisvollen Freunden durch das erste Jahr
}

\author{
Nach zwei Jahren Vorbereitungszeit und ein Jahr nach der Neueröffnung meiner Praxis macht es Sinn, ein \\ erstes kleines Resumée zu ziehen. Und das lautet: Im ersten Jahr ist Einsatz gefragt!
}

\author{
Dr. Philipp F. Gebhardt // \\ BISS45 - Kieferorthopädie Berlin \\ www.biss45.de \\ Dr. Gebhardt berichtet in jeder Ausgabe \\ über seinen Weg vom abgeschlossenen \\ Studium zum jungen Zahnarzt.
}
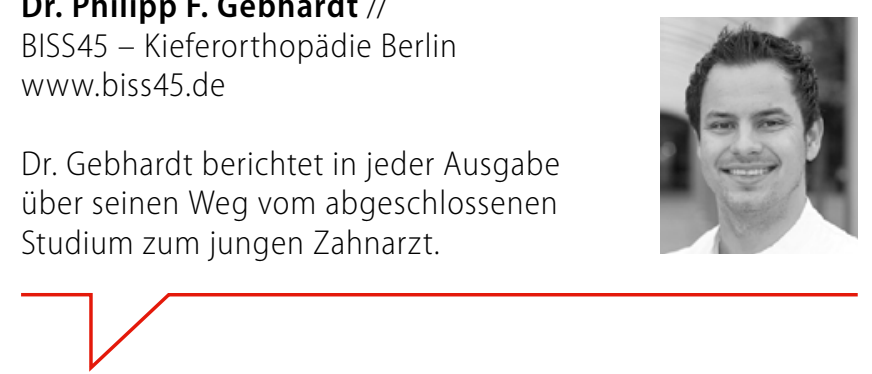

Nachdem ich in den letzten Ausgaben über die komplizierte Personalsuche und der in den letzten Jahren immer angespannteren Lage auf dem Arbeitsmarkt berichtet habe, stelle ich nun fest: Der anfängliche Fachkräftemangel hatte dann doch den Vorteil, dass die laufenden Kosten gering gehalten wurden. Zwar ist es wahrscheinlich möglich, durch hohe Werbe- und Marketingkosten direkt am Anfang einen hohen Patientendurchlauf $\mathrm{zu}$ generieren. Ich würde allerdings nicht davon ausgehen, dass dieser sich von seiner Qualität proportional zu den Investitionen verhält. Ein nachhaltiges Wachstum finde ich deshalb äußerst wichtig, um effiziente Abläufe zu etablieren. Direkt nach einer Neugründung ist es also sinnvoll, gezielt zu investieren und vor allem private Ausgaben und Nebenkosten möglichst gering zu halten. Ein detaillierter Businessplan und ein guter Steuerberater können hier vor Fehlinvestitionen und komplizierten Nachfinanzierungen schützen.

Allerdings sind nicht unbedingt die offensichtlichen Fehler wie eine Sportwagenfinanzierung in den ersten drei Jahren die Gefahr. Es ist eher die Summe von multiplen, nicht eingeplanten Faktoren, die zu einem Problem werden können. Beispiele sind schlecht verhandelte Dispozinsen, ein zu früh gezogener Kredit mit Bereitstellungszinsen, sehr kurze Kreditlaufzeiten mit hohen Tilgungen, einige 1000 Euro mehr für eine größere Mietwohnung oder eine Immobilienfinanzierung. Wenn hier auch noch hohe Werbekosten und ein oder zwei Helferinnengehälter hinzukommen (die durch eigene Überstunden vermieden werden könnten), dann entstehen schnell monatliche(!) Zusatzkosten im 5-stelligen Bereich. Man sollte für eine Neugründung also definitiv für das erste Jahr 7-Tage-Wochen mit mindestens zwölf Arbeitsstunden am Tag einplanen, um eine gute Kostenkontrolle zu gewährleisten. Des Weiteren macht es auch Sinn, sein soziales Umfeld vorher richtig einzuschätzen, um zumindest privaten Stressmomenten vorzubeugen. Ich bin momentan froh, nach einem Jahr ohne Engpässe schon wieder eine 40-50-Stunden-Woche zu haben und glaube zumindest, dass die meisten Freunde verstehen, dass ich mich seit der Einweihungsparty noch nicht wieder gemeldet habe.

\section{Impressum}

Eigentümer \& Copyright @ Springer-Verlag Berlin Heidelberg 2015, Springer Medizin c/o Springer-Verlag GmbH, Tiergartenstr. 17, 69121 Heidelberg, Tel. +49 6221/487-0 Springer Medizin ist ein Teil der Fachverlagsgruppe Springer Science+Business Media Geschäftsführung Springer Medizin:

Joachim Krieger (Managing Director Professional Group Springer Nature), Fabian Kaufmann

Director Apotheke und Zahnmedizin: Marion Bornemann Redaktionsleitung "der junge zahnarzt":

Dr. Swanett Koops (Tel. -8217, swanett.koops@springer.com)

Redaktion CME-Rubrik: Dr. Swanett Koops (Tel. -8217, swanett.koops@springer.com)

Layout: Arnulf Illing (Tel. -8952, arnulf.illing@springer.com)

Bildredaktion: Christiane Seufert

Anzeigenleitung: Marita Säuberlich (Tel. -8309, Fax -68309,

marita.saeuberlich@springer.com)

Corporate Publishing: Anja Weisse (Tel. -8739, Fax -68739, anja.weisse@springer.com)

Druck: PHOENIX PRINT GmbH, Würzburg. Printed in Germany

Erscheinungsweise: 4 Ausgaben pro Jahr

Papierausgabe: ISSN 1869-5744, gedruckt auf säurefreiem Papier

Bezugspreise (unverb. Preisempfehlung inkl. 7\% deutscher MwSt. und Versand): Vor-

zugspreis für persönliche Abonnenten: EUR 56,-; Einzelheftpreis: EUR 22,-. Das Abonne-

ment kann jederzeit 2 Monate vor Ende des Bezugszeitraumes gekündigt werden. Bestel-

lungen oder Rückfragen nimmt jede Buchhandlung oder der Verlag entgegen.
Kontakt: Springer Customer Service Center GmbH, Haberstr. 7, 69126 Heidelberg, Tel. +49 6221/345-4303, Fax-4229, Leserservice@springer.com (Mo.-Fr. 8.00 Uhr bis 20.00 Uhr) Copyright \& allgemeine Hinweise: Die Zeitschrift sowie alle in ihr enthaltenen einzelnen Beiträge und Abbildungen sind urheberrechtlich geschützt. Jede Verwertung, die nicht ausdrücklich vom Urheberrechtsgesetz zugelassen ist, bedarf der vorherigen schriftlichen Zustimmung des Verlags. Das gilt insbesondere für Vervielfältigungen, Bearbeitungen, Übersetzungen, Mikroverfilmungen und die Einspeicherung und Verarbeitung in elektronischen Systemen.

Gezeichnete Artikel geben nicht unbedingt die Meinung der Redaktion wieder. Autoren können unter bestimmten Voraussetzungen an der Ausschüttung der Bibliotheks- und Fotokopietantiemen teilnehmen. Einzelheiten bei VG WORT, Abt. Wissenschaft, Goethestr. 49, 80336 München.

Angaben über Dosierungsanweisungen und Applikationsformen sind anhand anderer Literaturstellen oder der Packungsbeilage auf ihre Richtigkeit zu überprüfen. Der Verlag übernimmt keine Gewähr.

Der IVW (Informationsgemeinschaft zur Feststellung der Verbreitung von Werbeträgern) angeschlossen. 